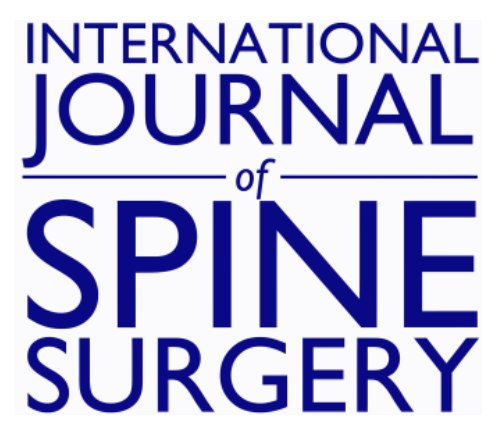

\title{
Development of Stabilimax NZ From Biomechanical Principles
}

\author{
Manohar M. Panjabi and Jens Peter Timm \\ Int J Spine Surg 2007, 1 (1) 2-7 \\ doi: https://doi.org/10.1016/SASJ-2006-0006-CO \\ http://ijssurgery.com/content/1/1/2
}

This information is current as of April 26, 2023.

Email Alerts Receive free email-alerts when new articles cite this article. Sign up at: http://ijssurgery.com/alerts 


\title{
Development of Stabilimax NZ From Biomechanical Principles
}

Manohar M. Panjabi, PhD, and Jens Peter Timm, BS

\begin{abstract}
Background

Traditionally, spinal degeneration and injury have been associated with abnormal intervertebral motion; thus, treatment for lowback pain has centered on prevention of motion through spinal fusion. Although the rate of successful spinal fusions is improving, complications such as adjacent-level syndrome emphasize the need to develop alternatives for treating spinal degeneration. In an effort to improve the clinical outcomes associated with such treatment, we hypothesized that spinal stabilization and a consequent reduction in symptoms is achievable without the harsh restrictions to spinal motion imposed by fusion. This idea was based on the principle of the neutral zone and the neutral zone hypothesis of back pain.
\end{abstract}

\section{Development}

Performance requirements for a novel device were determined through a series of biomechanical experiments. From these data, the Stabilimax NZ was developed to provide stabilization to a degenerated or surgically destabilized spine while maintaining the maximum possible total range of motion. Applied Spine Technologies Inc has tested 70 bilateral assemblies of the final design of the Stabilimax NZ, and all exceeded the biomechanical, static, fatigue, wear, and histological requirements necessary to initiate clinical investigation.

\section{Discussion}

The Stabilimax NZ device has been systematically designed and tested under protocols developed by Applied Spine Technologies in conjunction with Panjabi, Patwardhan, and Goel. The device decreased the neutral zone in destabilized spines while maintaining substantial range of motion.

\section{Clinical Relevance}

Development testing has been submitted to the US Food and Drug Administration and permission obtained to initiate an investigational device exemption trial to clinically investigate the efficacy of the Stabilimax NZ device.

Key Words neutral zone, dynamic stabilization. SAS Journal. Winter 2007;1:2-7. DOI: SASJ-2006-0006-CO

\section{INTRODUCTION}

This commentary is a review of the authors' hypothesis of back pain and the subsequent development of a dynamic stabilization device based on this hypothesis. Because the device is currently undergoing clinical investigation in an IDE trial, no conclusive results are available to report. A summary of the development efforts undertaken to date is provided here.

At some point in their lives, approximately $80 \%$ of adults will suffer acute back pain as a result of the normal degenerative process of the spine. ${ }^{1}$ As symptoms progress, lumbar surgery may become an option for treatment. In fact, doctors perform more than 200,000 spinal fusion operations each year in the United States to address severe physical disability associated with low-back pain. Two thirds of these procedures are performed in the lumbar spine. ${ }^{2,3}$

\section{BACKGROUND}

Albee pioneered lumbar spinal fusion in 1911 to treat degenerative spinal disease. ${ }^{4}$ In 1933, Burns introduced the anterior lumbar interbody fusion technique. ${ }^{5}$ Later that decade, metallic implants were first utilized to provide supplemental fixation for fusion procedures. Recently, with the advent of bone morphogenetic proteins, successful fusion rates have 
approached $100 \%$. Despite the progress over the past 70 years in the surgical technique for spinal fusion, the effectiveness of lumbar fusion to decrease pain and improve functioning has yielded mixed results when assessed through long-term follow-up of clinical outcomes.

In 1960, Harmon published 10-year follow-up results of lumbar disc excision and fusion in 30 patients. ${ }^{6}$ Harmon reported an osseous union (fusion) rate of $82.8 \%$ and an unsatisfactory clinical outcome in 3 of the 30 patients, a $90 \%$ success rate. More than 30 years later, in 1994, Mardjetko performed a metaanalysis of studies of lumbar fusion outcomes. He reported that posterior fusion without instrumentation produced fusion rates of $86 \%$ and a satisfactory clinical outcome of $90 \%$. $^{7}$ These findings are similar to those yielded by Yuan's meta-analysis of pedicle screw fusion outcomes. This study, also published in 1994, reported a $93 \%$ fusion rate and an $86 \%$ satisfaction rate when decompression and fusion included pedicle screw instrumentation. ${ }^{8}$ When compared with Harmon's surgical results, Mardjetko's and Yuan's recent studies highlight the fact that significant improvements in fusion rates have not translated to equivalent improvements in patient outcomes or satisfaction.

Long-term clinical outcomes of lumbar fusion have not reflected the significant progress made in technique and instrumentation. One explanation for the discrepancy lies in the effect that the surgical procedure has on spine biomechanics. When a vertebral joint is permanently fused, the vertebral levels adjacent to the fused joint are subjected to additional loads and stresses during routine activities. ${ }^{9}$ Adjacent-level syndrome occurs when the spinal joints adjacent to the site of the original fusion degenerate. It is a potential side effect of lumbar fusion that may result in the need for additional surgery. MacDougall's 2003 study of 178 patients with a minimum 10-year followup reported an $18 \%$ rate of adjacent segment degeneration, requiring subsequent surgery. ${ }^{10}$ In addition, only $70 \%$ of the patients surveyed judged their initial surgery to be a success 10 years later. Although the rate of successful fusions may be improving, complications such as adjacent level syndrome, as reported by MacDougall and others, emphasize the need to develop alternatives for treating spinal degeneration. ${ }^{11-13}$

\section{HYPOTHESIS}

In an effort to improve the clinical outcomes associated with such treatment, we hypothesized that spinal stabilization and a consequent reduction in symptoms is possible without the harsh restrictions to spinal motion imposed by fusion. This idea is based on the principle of the neutral zone and the neutral zone hypothesis of back pain.

\section{THE NEUTRAL ZONE}

One consequence of both tissue degeneration and surgical injury is abnormal intervertebral motion, which may be associated with pain in the lumbar spine. The change in spinal motion associated with spinal injury has been evaluated in a series of biomechanical studies. We defined the neutral zone as a region of intervertebral laxity around neutral posture. ${ }^{14}$ In a series of published articles, Panjabi evaluated the effects of injury, degeneration, and stabilization on the neutral zone of the spine. ${ }^{14-18}$ The neutral zone is very sensitive to changes in the spinal tissues and is therefore a clinically important measure of spinal instability. These evaluations, along with continual investigation into the interconnection of spinal biomechanics and clinical symptoms, has led to the neutral zone hypothesis of back pain. ${ }^{19-21}$ This hypothesis has, in turn, been used to provide guidance for the development of an alternative means of spinal stabilization.

This alternative method is based on the hypothesis that an increase in the neutral zone (laxity of the spine around neutral posture) because of injury or degeneration causes a cascade of effects that ultimately results in increased loading of various spinal components, accelerated degeneration, and back pain. ${ }^{22}$ To develop successful alternatives for treating spinal disorders, we investigated the interaction between the biomechanics of the spine and the mechanical parameters of the device.

To understand the neutral zone principle, it is necessary to understand the 3 subsystems of spinal stability ${ }^{19}$ : the passive

Figure 1

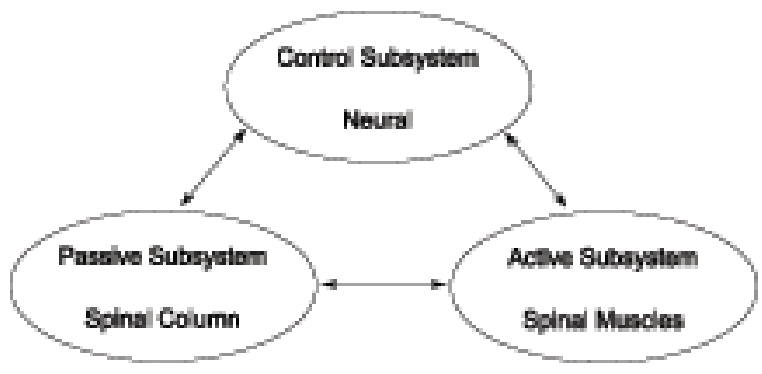

Biomechanical Components of the Spinal Stabilizing System

The 3 subsystems of the spinal stability system. ${ }^{19}$

subsystem (spinal column), the active subsystem (spinal muscles), and the control subsystem (neural) (Figure 1). Under normal conditions, these 3 subsystems maintain the mechanical stability of the spine. Damage or dysfunction in one subsystem requires the other 2 subsystems to compensate. 
Early studies focused on the effect of spinal injury on the stability of the spinal column (passive subsystem). The removal of a disc's nucleus produced an increase in flexion, lateral bending, and axial rotation. ${ }^{15}$ Other researchers, using similar models of spinal injury, have also observed that degeneration and trauma produced multidirectional laxity in the spinal column. ${ }^{23}$

The contribution of the spinal muscles (active subsystem) to the stability of the spine has also been investigated. After each injury, simulated muscle forces applied to the spinous process reduced the neutral zone to almost its intact value, without significantly affecting ROM. ${ }^{24}$ This finding has been supported by other research, ${ }^{25}$ and the results suggest that muscles in an injured spine perform extra work predominantly to restabilize the neutral zone, not to restrict the overall ROM.

The third spinal stabilizing subsystem (neuromuscular control) has been evaluated by comparing cohorts of patients with back pain to patients without back pain. Marras has demonstrated that there is a higher level of muscle activity in those patients experiencing back pain. ${ }^{26}$ It is theorized that this increase in muscle activity is triggered by the neural subsystem's responding to maintain the needed mechanical stability not being provided by the degenerated or injured passive subsystem. When muscles are recruited to compensate for the laxity of the spine, dysfunction and low-back pain appear to result. ${ }^{20}$

Traditionally, spinal degeneration and injury have been associated with abnormal intervertebral motion, which meant that the treatment for low-back pain was centered on the prevention of motion through spinal fusion. A review of the available literature reveals conflicting data about the relationship between an increase in spinal range of motion and the development of low-back pain. ${ }^{27-30}$

To examine the effect of a fixation system on the neutral zone and total ROM, we applied an external fixator to an isolated cervical spine preparation. The fixator decreased the neutral zone by $68.8 \%$, substantially greater than the $39.3 \%$ decrease observed in ROM. ${ }^{18}$ This may indicate that when back injury occurs, the neutral zone is increased to a greater extent than the ROM; in a similar manner, the fixation of the spine decreases the neutral zone to a greater extent than it decreases ROM. Thus, change in the neutral zone may be a more significant indicator of spinal stability/instability than the ROM. ${ }^{20}$ This principle provided the framework for the development of a device that preferentially decreased the neutral zone while substantially maintaining physiological ROM.

\section{DEVICE DEVELOPMENT}

Pedicle screw-based dynamic stabilization systems are surgical devices that are implanted in the posterior spine. These devices work by preserving flexibility between the lumbar vertebrae while adding stability to the affected joints. The dynamic stabilization devices currently available have been developed in a variety of ways. The authors conceived a device that would be designed with mechanical properties derived through iterative experimental validation to have a specific predetermined effect on spine biomechanics. Thus far, it appears that this novel method of directly linking a hypothesis of back pain to the functionality of a stabilization device, and then designing it to experimentally determined criteria, is unique.

The design of this device was undertaken with keen interest in 3 areas of development:

- Providing stabilization to a degenerative or surgically destabilized spine while maintaining the maximum possible range of spinal motion. Stabilization has been defined as reduction of the neutral zone to sub-intact levels and preservation of total motion at approximately $80 \%$ of intact levels.

- Designing an associated instrument system compatible with minimally destructive surgical methods, such as the Wiltse technique, to maintain the innate stabilizing capacity of the muscles. ${ }^{31,32}$

- Verifying suitable device fatigue strength, wear resistance and bio-compatibility through validated testing protocols.

In conjunction with Panjabi, Applied Spine Technologies Inc. (New Haven, Conn) has developed a device, the Stabilimax $\mathrm{NZ}$, in accordance with the neutral zone principle of spinal biomechanics. The device, a posterior pedicle screw-based dynamic stabilization system, features dual concentric springs combined with a ball-and-socket joint on each end. The device is designed to enhance the resistance of the passive spinal system around neutral posture (the neutral zone) while maintaining the maximum possible range of motion.

\section{EXPERIMENTAL VERIFICATION}

Panjabi conducted initial development experimentation to determine optimal device parameters for spring stiffness and associated interpedicular travel. Patwardhan, Goel, and Panjabi conducted subsequent studies to verify the effect of the device following progressive destabilization procedures. Applied Spine Technologies Inc and several contract facilities performed static, fatigue, wear, and in vivo histologic evaluation to demonstrate that the device met strength, durability, and compatibility requirements.

The characteristics of the device were optimized through a set of biomechanical studies. Optimal spring stiffness for the device was determined in both the intact spine and the spine destabilized by nucleotomy and laminectomy with partial 
facetectomy. Optimal interpedicular travel of the device was established for both compression of the device during spinal extension and elongation of the device during spinal flexion. Optimal spring stiffness of $90 \mathrm{~N} / \mathrm{mm}$ and associated travel were determined when the device was capable of consistently reducing the neutral zone to the pre-destabilized levels regardless of specimen condition while maintaining the maximum possible range of spinal motion.

Patwardhan tested 6 human cadaveric L2-L5 specimens to determine the effect of the Stabilimax NZ (Figure 2) on range of motion, neutral zone, and axis of rotation of the spine. The flexibility protocol with both a $0 \mathrm{~N}$ and $400 \mathrm{~N}$ follower load was applied for flexion/extension, lateral bending, and axial rotation, as previously described by Patwardhan et al. ${ }^{33}$ Specimens were tested (1) intact, (2) after destabilization consisting of L3 laminectomy, bilateral L3-L4 foraminotomy, and L3-L4 nucleotomy, and (3) after implantation of Stabilimax NZ at L3L4. Destabilization showed an increase in ROM and an increase in the neutral zone. With the application of Stabilimax NZ, the range of motion and neutral zone of the instrumented level were reduced, stabilizing the motion of the spine. The axis of rotation, measured from fluoroscopic images taken at maximum rotations as previously described by Patwardhan, ${ }^{34}$ demonstrated a 1.9$\mathrm{mm}$ posterior shift from comparable intact axis of rotation with

Figure 2

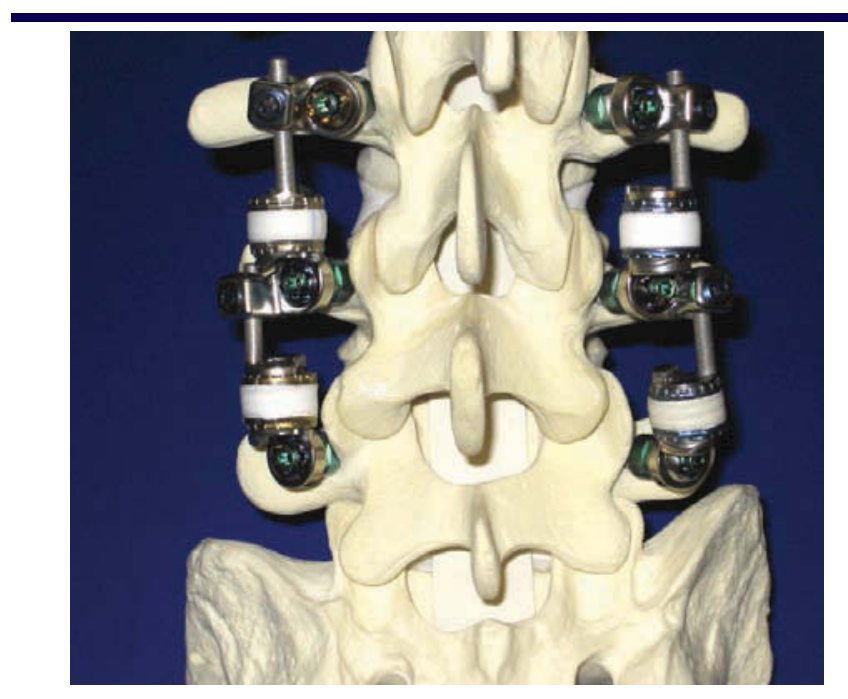

Two-level Stabilimax NZ.

a standard deviation of $1.6 \mathrm{~mm}$. It is important to note that more motion was maintained after instrumentation with the Stabilimax $\mathrm{NZ}$ than has been found with fusion constructs. ${ }^{35,36}$ (A more detailed analysis of this data has been accepted for presentation at the 2007 Annual Meeting of the International Society for the Study of the Lumbar Spine.)

Goel performed a verification study using his previously validated finite element model of the ligamentous lumbar spine, levels L3-S1, instrumented with the Stabilimax NZ. The validity of the finite element model analysis was supported by a strong correlation between the motions predicted by the finite element spinal model and the in vitro cadaver experiments previously conducted by Patwardhan. We used this model to evaluate the complex loading on internal elements of the device and spinal tissues otherwise impossible to quantify. The finite element model analysis demonstrated that the Stabilimax NZ device provided approximately twice the motion compared with a typical fusion system in response to moments placed on the superior vertebra. Also, with preload, maximum Von Mises stress observed in the pedicle screw was $121 \mathrm{MPa}$ with the rigid fusion system, compared to $36 \mathrm{MPa}$ with the Stabilimax NZ. (A more detailed analysis of this data has been accepted for presentation at the 2007 Spine Arthroplasty Society Annual Meeting.)

Panjabi performed cadaveric testing on 6 human lumbar spine sections, T12-S1, under the flexibility and hybrid testing protocols. This testing provided a direct comparison to a fusion construct at the implanted level and also evaluated the comparative adjacent level effect in the spine specimens with fusion and with Stabilimax NZ. Following intact testing, the spine was destabilized by a total laminectomy at L4 plus a bilateral partial facetectomy at L4-L5. After implementation of the Stabilimax NZ at the L4-L5 level, the motion of the spine was stabilized in flexion/extension and lateral bending. The average adjacent-level effect in the form of increased motion for Stabilimax NZ was approximately half that for fusion (unpublished data).

Performance requirements for the Stabilimax NZ have been based on the long clinical history of pedicle screw fusion systems and biomechanical data. We tested 70 bilateral assemblies of the final design to demonstrate the safety of the device, and all exceeded static, fatigue, wear resistance, including particle characterization, and histological requirements. We have submitted all development testing to the US Food and Drug Administration and obtained permission to initiate an IDE trial to clinically investigate the efficacy of the Stabilimax NZ device.

In an effort to improve on the long-term clinical outcomes yielded by current fusion technology, Panjabi has developed a dynamic spine stabilization system based on the neutral zone hypothesis of back pain. Under protocols developed by Applied Spine Technologies in conjunction with Panjabi, Patwardhan, and Goel, the Stabilimax NZ device has been systematically developed and tested. The device decreased the neutral zone in destabilized spines while maintaining substantial range of motion. Additionally, we have evaluated the device rigorously to demonstrate its strength, durability, and biocompatibility required for clinical use. The Stabilimax NZ provides a new alternative for the treatment of spinal degeneration, one that invites further investigation. 
Manohar M. Panjabi, PhD, Jens Peter Timm, BS

From the Department of Orthopaedics and Rehabilitation, Yale University School of Medicine, New Haven, Conn (Panjabi); and Applied Spine Technologies Inc, New Haven (Timm).

Manohar M. Panjabi is cofounder and a paid consultant of Applied Spine Technologies Inc, New Haven, Conn. Jens Peter Timm is an employee of Applied Spine Technologies Inc.

Address correspondence and reprint requests to Manohar M. Panjabi, PhD, Biomechanics Research Laboratory, Department of Orthopaedics and Rehabilitation, Yale University School of Medicine, PO Box 208071, New Haven, CT 06520-8071 (email: manoharpanjabi@gmail.com).

This submission was received January 3, 2007, and accepted for publication February 26, 2007.

Institutional review board approval was not required.

\section{REFERENCES}

1. Nachemson A. The lumbar spine: an orthopaedic challenge. Spine. 1976;1:59-71.

2. Health Research International. Opportunities in US Spinal Fusion Markets. Cleveland, Ohio: Health Research International; 2003. Report 104-1-US-0203.

3. Owings MF, Kozak LJ. Ambulatory and inpatient procedures in the United States, 1996. National Center for Health Statistics. Vital Health Stat 12(139). 1998.

4. Albee FH. Transplantation of a portion of the tibia into the spine for Pott's disease. A preliminary report. Journal of A.M.A. 1911; 57:885-886.

5. Burns BH. An operation for Spondylolisthesis. The Lancet. $1933 ; 221: 1233$

6. Harmon E. Anterior extraperitoneal lumbar disk excision and vertebral body fusion. Clin Orthop. 1960;18:169-198.

7. Mardjetko SM, Connolly PJ, Shott S. Degenerative lumbar spondylolisthesis: a meta-analysis of literature 1970-1993. Spine. 1994; 19:2256S-2265S.

8. Yuan HA, Garfin SR, Dickman CA, Mardjetko SM. A historical cohort study of pedicle screw fixation in thoracic, lumbar, and sacral spinal fusions. Spine. 1994;19:2279S-2296S.

9. Lee CK, Langrana NA. Lumbosacral spinal fusion: a biomechanical study. Spine. 1984;9:574-581.
10. MacDougall J. Incidence of adjacent segment degeneration at ten years after lumbar spine fusion. Presented at: 18th Annual Meeting of the North American Spine Society, October 21-25, 2003, San Diego, Calif.

11. Gillet $\mathrm{P}$. The fate of the adjacent motion segments after lumbar fusion. J Spinal Disord Tech. 2003;16:338-345.

12. Ishihara H, Osada R, Kanamori M, et al. Minimum 10-year follow-up study of anterior lumbar interbody fusion for isthmic spondylolisthesis. J Spinal Disord. 2001;14:91-99.

13. Kumar MN, Jacquot F, Hall H. Long-term follow-up of functional outcomes and radiographic changes at adjacent levels following lumbar spine fusion for degenerative disc disease. Eur Spine J. 2001;10:309-313.

14. Panjabi MM, Goel VK, Takata K. Physiologic strains in the lumbar spinal ligaments: an in vitro biomechanical study 1981 Volvo Award in Biomechanics. Spine. 1982;7:192-203.

15. Panjabi MM, Krag MH, Chung TQ. Effects of disc injury on mechanical behavior of the human spine. Spine. 1984;9:707713.

16. Panjabi MM, Abumi K, Duranceau J, Crisco JJ. Biomechanical evaluation of spinal fixation devices II: Stability provided by eight internal fixation devices. Spine. 1988;13:1135-1140.

17. Panjabi MM, Oxland TR, Yamamoto I, Crisco JJ. Mechanical behavior of the human lumbar and lumbosacral spine as shown by three-dimensional load-displacement curves. J Bone Joint Surg Am. 1994;76:413-424.

18. Panjabi MM, Lydon C, Vasavada A, Grob D, Crisco JJ III, Dvorak J. On the understanding of clinical instability. Spine. 1994;19:2642-2650.

19. Panjabi MM. The stabilizing system of the spine. Part I. Function, dysfunction, adaptation, and enhancement. J.Spinal Disord. 1992;5:383-389.

20. Panjabi MM. The stabilizing system of the spine. Part II. Neutral zone and instability hypothesis. J.Spinal Disord. 1992;5:390396.

21. Panjabi MM. Clinical spinal instability and low back pain. $J$ Electromyogr Kinesiol. 2003;13:371-379.

22. Panjabi MM. A hypothesis of chronic back pain: ligament subfailure injuries lead to muscle control dysfunction. Eur Spine J. 2006; 15:668-676.

23. Goel VK, Nishiyama K, Weinstein JN, Liu YK. Mechanical properties of lumbar spinal motion segments as affected by partial disc removal. Spine. 1986;11:1008-1012. 
24. Panjabi MM, Duranceau JS, Oxland TR, Bowen CE. Multidirectional instabilities of traumatic cervical spine injuries in a porcine model. Spine. 1989;14:1111-1115.

25. Kettler A, Hartwig E, Schultheiss M, Claes L, Wilke HJ. Mechanically simulated muscle forces strongly stabilize intact and injured upper cervical spine specimens. J Biomech. 2002;35:339346.

26. Marras WS, Davis KG, Ferguson SA, Lucas BR, Gupta P. Spine loading characteristics of patients with low back pain compared with asymptomatic individuals. Spine. 2001;26:2566-2574.

27. Dvorak J, Panjabi MM, Novotny JE, Chang DG, Grob D. Clinical validation of functional flexion-extension roentgenograms of the lumbar spine. Spine. 1991;16:943-950.

28. Friberg O. Lumbar instability: a dynamic approach by tractioncompression radiography. Spine. 1987;12:119-129.

29. Vanharanta H, Sachs B.L, Spivey MA, et al. The relationship of pain provocation to lumbar disc deterioration as seen by CT/ discography. Spine. 1987;12:295-298.

30. Pearcy M, Portek I, Shepherd J. The effect of low-back pain on lumbar spinal movements measured by three-dimensional X-ray analysis. Spine. 1985;10:150-153.

31. Wiltse LL, Jackson DW. Treatment of spondylolisthesis and spondylolysis in children. Clin Orthop Relat Res. 1976;92-100.

32. Vialle R, Wicart P, Drain O, Dubousset J, Court C. The Wiltse paraspinal approach to the lumbar spine revisited: an anatomic study. Clin Orthop Relat Res. 2006;445:175-180.

33. Patwardhan AG, Havey RM, Meade KP, Lee B, Dunlap B. A follower load increases the load-carrying capacity of the lumbar spine in compression. Spine. 1999;24:1003-1009.

34. O'Leary P, Nicolakis M, Lorenz MA, et al. Response of Charité total disc replacement under physiologic loads: prosthesis component motion patterns. Spine J. 2005;5:590-599.

35. Phillips FM, Cunningham B, Carandang G, et al. Effect of supplemental translaminar facet screw fixation on the stability of stand-alone anterior lumbar interbody fusion cages under physiologic compressive preloads. Spine. 2004;29:1731-1736.

36. Renner SM, Voronov L, Ghanayem A, et al. Biomechanical stability of isthmic spondylolisthesis with posterolateral and circumferential fusion. Presented at: 52nd Orthopaedic Research Society Annual Meeting, March 19-22, 2006, Chicago, Ill. 